\title{
Resilience of state-of-the-art 1300nm In(Ga)As/GaAs quantum-dot lasers to external optical feedback (Withdrawal Notice)
}

Soroush Sobhani, David Childs, Keizo Takemasa, Kenichi Nishi, Mitsuru Sugawara, et al.

Soroush A. Sobhani, David T. Childs, Keizo Takemasa, Kenichi Nishi, Mitsuru Sugawara, Richard A. Hogg, "Resilience of state-of-the-art 1300nm In (Ga)As/GaAs quantum-dot lasers to external optical feedback (Withdrawal Notice)," Proc. SPIE 10946, Metro and Data Center Optical Networks and Short-Reach Links II, 1094609 (1 February 2019); doi: 10.1117/12.2509931 


\section{Resilience of state-of-the-art 1300nm In(Ga)As/GaAs quantum-dot lasers to external optical feedback (Withdrawal Notice)}

Soroush A. Sobhani, David T. Childs, and Richard A. Hogg

Univ. of Glasgow (United Kingdom)

Keizo Takemasa, Kenichi Nishi, and Mitsuru Sugawara

QD Laser, Inc. (Japan)

Proc. SPIE 10946, 1094609 (2019)

Online Publication Date: 1 February 2019

Withdrawn from Publication: 15 March 2019

Conference Date: 2-7 February 2019

Conference Location: San Francisco, California, United States

Conference Title: Metro and Data Center Optical Networks and Short-Reach Links II

Conference Chairs: Atul K. Srivastava, Madeleine Glick and Youichi Akasaka

Publisher's Note: This paper was published in error on 1 February 2019 by the publisher and was withdrawn on 15 March 2019. SPIE regrets this error. 